\title{
OPEN A network meta-analysis of the dose-response effects of lurasidone on acute schizophrenia
}

\author{
Manit Srisurapanont ${ }^{1 凶}$, Sirijit Suttajit ${ }^{1}$, Surinporn Likhitsathian ${ }^{1}$, Benchalak Maneeton ${ }^{1}$ \& \\ Narong Maneeton ${ }^{1}$
}

We compared the efficacy, safety, and acceptability of lurasidone at different doses to establish the dose-response relationships of lurasidone therapeutic and adverse effects in acute schizophrenia. Included trials were 4- to 16-week, fixed-dose, randomized controlled trials of lurasidone in adults with acute schizophrenia. Different doses of lurasidone, other antipsychotics, and placebo were considered as independent treatments. Apart from all-cause dropout rates, four therapeutic and four adverse outcomes were included in the frequentist network meta-analysis (NMA). Lurasidone 160, $120,80,40$, and $20 \mathrm{mg} /$ day were studied in ten trials of 3,366 adults with schizophrenia exacerbation. Lurasidone $160 \mathrm{mg} /$ day reduced Positive and Negative Syndrome Scale (PANSS) total scores significantly more than lurasidone 120, 80, 40, and $20 \mathrm{mg}$ /day (mean differences = - 7.63, - 7.04, - 8.83, and - 12.25, respectively). All-cause dropout rates were significantly lower in participants receiving lurasidone $160 \mathrm{mg} /$ day and $80 \mathrm{mg} /$ day compared with those taking placebo. The halfmaximal effective doses of lurasidone for PANSS total, PANSS positive, and MADRS score reductions were higher than $80 \mathrm{mg} /$ day. The confidence of all NMA estimates was low or very low. Lurasidone $160 \mathrm{mg} /$ day is currently the most efficacious and acceptable dose for acute schizophrenia. Its maximal effective doses may be higher than $160 \mathrm{mg} /$ day.

Behavioral symptoms of schizophrenia are complex, and each syndrome may respond to different doses of an antipsychotic. Among the heterogeneous symptoms of schizophrenia, common syndromes include positive, negative, and depression syndromes ${ }^{1}$. The doses of quetiapine and aripiprazole effective for negative symptoms are more narrow than those effective for overall psychotic and positive symptoms ${ }^{2,3}$. These findings suggest that the dose-response relationships of antipsychotics may vary among schizophrenia syndromes. The findings of two pairwise meta-analyses (PMAs) suggested that the approved doses of 40-160 mg/day are effective for various syndromes of schizophrenia, as effective as most antipsychotics, well-tolerated, and less likely to cause weight gain ${ }^{4,5}$.

The evidence so far suggests that lurasidone doses and effects for acute schizophrenia may be inconsistent. Lurasidone $80 \mathrm{mg} /$ day was not effective for acute schizophrenia in some randomized trials $\mathrm{s}^{6-8}$ but outperformed both placebo and lurasidone $120 \mathrm{mg} /$ day in another trial ${ }^{9}$. A recent network meta-analysis (NMA) found that lurasidone $40 \mathrm{mg} /$ day increased body weight, but lurasidone $80 \mathrm{mg} /$ day was associated with weight loss ${ }^{10}$. The product monograph of lurasidone informed that many adverse effects of lurasidone $120 \mathrm{mg} /$ day are more common than those of lurasidone $160 \mathrm{mg} /$ day, e.g., somnolence ( $26 \%$ vs. $8 \%)$, akathisia $(22 \% \text { vs. } 7 \%)^{11}$. These findings suggest an inconsistent ordering of lurasidone doses and effects. The dose adjustment of lurasidone for treating acute schizophrenia is, therefore, challenging.

Little has been known about the dose-response relationships of lurasidone for schizophrenia syndromes and its common side effects. One NMA compared the clinical effects of only three treatments of lurasidone $80 \mathrm{mg} /$ day, lurasidone $40 \mathrm{mg} /$ day, and placebo. This NMA found that lurasidone 80 and $40 \mathrm{mg} /$ day reduced most psychopathology to a similar extent ${ }^{10}$. A PMA compared the effects of the minimal effective dose (MED) of lurasidone with its 2- and threefold MEDs ${ }^{12}$. The 2- and threefold MEDs of lurasidone were superior to its MED in reducing overall and positive psychotic symptoms but not negative symptoms. Only one dose-response meta-analysis of lurasidone has been conducted. This meta-analysis used a multivariate statistical technique to

\footnotetext{
${ }^{1}$ Department of Psychiatry, Faculty of Medicine, Chiang Mai University, Si Phum, 110 Inthawarorot Road, Mueang 50200, Chiang Mai, Thailand. ${ }^{2}$ These authors contributed equally: Manit Srisurapanont and Sirijit Suttajit. 范mail: manit.s@cmu.ac.th
} 
synthesize the data, construct the dose-response curve, and estimate the effective dose of lurasidone for overall psychotic symptoms only ${ }^{13}$. It found that the maximal effective dose of lurasidone might be higher than $160 \mathrm{mg} /$ day. The findings of these meta-analyses suggest that the dose-response relationships of lurasidone for many treatment effects remain unknown.

As an antipsychotic with variable treatment effects, the dose-response information of lurasidone treatment effects may guide its dose adjustment in managing schizophrenia syndromes and common adverse effects. This study aimed to compare the efficacy, safety, and acceptability of lurasidone at different doses to establish the dose-response relationships of lurasidone therapeutic and adverse effects in acute schizophrenia.

\section{Methods}

Protocol and registration. The protocol of this systematic review was prospectively registered at PROSPERO (CRD42020201337). The report of this NMA was based on the PRISMA 2015 Network Meta-Analysis Checklist (see STable 1) ${ }^{14}$. MS and SS independently screened the titles and abstracts, evaluated the full-text publications, selected the trials, extracted the data, and assessed the trial quality. If there was any discrepancy, these two reviewers resolved it using a consensus discussion.

Eligible criteria. Included trials were 4- to 16-week, fixed-dose, randomized controlled trials (RCTs) of lurasidone in adults with acute schizophrenia that reported at least one outcome of interest. Trials or trial arms with a cross-over study design or examining the flexible doses of lurasidone were excluded. The data from trials or the trial arms that titrated the study medications in the first week could be included.

Age, sex, and severity of overall psychotic symptoms were considered as effect modifiers ${ }^{15}$. The medians and interquartile ranges of these modifiers were plotted to explore the transitivity among treatment groups.

Information sources, searches, and study selection. We searched Pubmed, Scopus, Web of Science, Cochrane Library, and ClinicalTrials.gov from the inception. Key search terms included "lurasidone" AND "schizophrenia". An additional limitation was applied by adding the term "random" or limiting to "randomizedcontrolled trial". The details of database searches can be found in STable 2. After the removal of duplicate records, we screened the titles/abstracts, evaluated the full-text publications, and selected the trials. No language restriction was applied for the study selection.

Data collection process and data extraction. We extracted the trial data using a data record form. Trial characteristics of interest included: i) study ID (first author, year); ii) participant characteristics, including age, diagnosis, percentage of male participants, the severity of overall psychotic symptoms, and study duration; iii) each fixed dose of lurasidone, other antipsychotics, and placebo; iv) the measures of psychotic and depression syndromes; v) adverse outcomes; and vi) dropout rates.

Data items. The primary outcomes of efficacy and acceptability were overall psychotic symptom reduction and all-cause dropout rates, respectively. Three secondary outcomes of efficacy included positive, negative, and depression symptom reduction. Four safety outcomes were weight gain, the incidence rates of somnolence, the incidence of extrapyramidal side effects (EPS), and dropout rates due to adverse events (or adverse dropout rates). The term "adverse dropout" used in this review referred to a participant who discontinued his/her assigned treatment, including placebo because he/she could not tolerate an adverse effect of that treatment. For each trial, we extracted only the last outcomes reported between 4 and 16 weeks.

Geometry of the network. All fixed doses of lurasidone, other antipsychotics, and placebo were considered as independent treatments. For a network plot, a treatment was drawn by a node, and a comparison between the treatments was shown by an edge. The edge thickness indicated the number of comparisons.

Risk of bias within individual trials. We assessed the trial quality using the revised tool to assess the risk of bias in randomized trials $(\operatorname{RoB} 2)^{16}$. This tool evaluated five domains of bias, including randomization processes, adherence to the assigned interventions, missing outcome data, the bias of measurement, and the bias of the reported results. Each domain was rated as low risk-of-bias, some concerns, or high risk-of-bias. The worst risk of bias in any of the domains was used for rating the overall risk of bias.

Summary measures, data analysis, and assessment of inconsistency. We computed a mean difference (MD) if the continuous outcome was measured using the same scale. Otherwise, that outcome would be computed as a standardized mean difference (SMD). We aggregated the dichotomous outcomes using relative risks (RRs).

For each pairwise comparison, the negative MD (or SMD) indicated the superiority of a lurasidone dose against the other lurasidone dose, the other antipsychotic, or placebo. We compared the efficacy, safety, and acceptability between treatments using a pairwise meta-analysis and among treatments using a frequentist NMA. All analyses were performed on a random-effect model. For each comparison, we reported direct, indirect, and NMA estimates. We described the treatment ranks using league tables.

We assessed the global heterogeneity (incoherence) of a dataset using the Cochran chi-square (Cochran Q) statistic incoherence tests ${ }^{17}$. The analysis of Separate Indirect from Direct Evidence (SIDE) was performed using the back-calculation method to identify the local inconsistency between a pair of direct and indirect estimates ${ }^{18}$. 
The inconsistency with the p-value between 0.05 and 0.10 or less than 0.05 was considered as some concern or major concern, respectively.

The data were analyzed and visualized using the netmeta version 1.2-1 package under the $R$ Program version 3.6 via the Rstudio software version 1.2.5 $5^{19-21}$.

Risks of bias across studies and additional analysis. We weighed the risks of bias using the sample sizes and plotted the risks of bias across trials. The publication bias was visualized using the funnel plots and quantified using the Egger's test $\mathrm{t}^{22,23}$.

Confidence in NMA estimates. Although this NMA included the data of other antipsychotics, their treatment effects and ranks were disregarded. This decision was made because we did not comprehensively search and include all RCT data of other antipsychotics.

We used a semiautomated software that assessed the confidence of NMA estimates based on the Confidence in NMA approach (CINeMA) ${ }^{24,25}$. Four grades of confidence used for rating an NMA estimate included high, moderate, low, or very low levels. The confidence of NMA estimates derived from RCTs was rated as high and was downgraded based on the following concerns: (1) within-study bias, (2) reporting bias, (3) indirectness, (4) imprecision, (5) heterogeneity, and (6) incoherence. Suspected reporting bias or major concern on any dimension were rated down by two levels. Some concern on a dimension resulted in the downgrading of confidence by one level. We summarized the risk of bias and indirectness using the majority.

Dose-response relationships. We performed graphic and quantitative exploration of dose-response relationships using the NMA estimates of lurasidone effects compared with those of placebo. Four therapeutic responses of interest included overall psychotic, positive, negative, and depression symptoms. Four adverse responses being examined were weight gain, somnolence, EPS, and adverse dropouts. Because all-cause dropout rates were the composite outcome of therapeutic and adverse effects, its dose-response relationship was not considered.

We plotted the doses and responses on the $\mathrm{x}$ - and $\mathrm{y}$-axes, respectively. The best-fit curve was plotted using the Hill equation, and the half-maximal effective dose (ED50) was calculated. The dose-response relationships were analyzed and visualized using Dr Fit software version $1.042^{26}$.

\section{Results}

Study selection. We searched the databases on July 29, 2020, and found 462 items (see Fig. 1). After the duplicate removal, 296 records remained for the title and abstract screening. We further assessed 15 full-text publications and excluded five trials, including two flexible-dose trials ${ }^{27,28}$, one 3-week trial ${ }^{29}$, one trial in adolescent patients $s^{30}$, and one trial in adults with treatment-resistant schizophrenia ${ }^{31}$. Finally, ten RCTs were included for network meta-analysis ${ }^{6-9,32-37}$.

Network structure and geometry. The NMA compared the efficacy, acceptability, and safety among ten independent treatments, including five doses of lurasidone (160,120,80,40, and $20 \mathrm{mg} /$ day), four antipsychotics (i.e., haloperidol, olanzapine, quetiapine, and risperidone), and placebo. Figure $2 \mathrm{~A}-\mathrm{H}$ show the network graphs of nine outcomes. The largest network was the dataset of Positive and Negative Syndrome Scale (PANSS) total score used for assessing the severity of overall psychotic symptoms (10 trials, 10 treatments, and 43 pairwise comparisons). The smallest network was the dataset of Montgomery-Asberg Depression Rating Scale (MADRS) scores used for assessing the severity of depression symptoms ( 5 trials, 9 treatments, and 29 pairwise comparisons). The networks of PANSS positive and negative scores were the same and included only four doses of lurasidone $(160,120,80$, and $40 \mathrm{mg}$ /day). All five doses of lurasidone (160, 120, 80, 40, and $20 \mathrm{mg} /$ day) were involved in the rest networks.

Study characteristics. After excluding two arms of lurasidone flexible doses ${ }^{33}, 3,366$ adults with acute schizophrenia of ten trials were included in this NMA (see Table 1). All were six-week trials. Nine trials had a placebo-controlled arm. Other antipsychotics and their doses (number of participants) were as follows: haloperidol $10 \mathrm{mg} /$ day $(n=72)$, olanzapine $10(n=51)$ and $15 \mathrm{mg} /$ day $(n=123)$, quetiapine $600 \mathrm{mg} /$ day $(n=120)$, and risperidone $4 \mathrm{mg} /$ day $(\mathrm{n}=64)$. Three age ranges of the participants included 18-75 years in six trials, 18-65 years in three trials, and 18-45 years in one triall ${ }^{32}$.

Mean age, sex, and severity of overall psychotic symptoms (based on PANSS total score) of each treatment were summarized and visualized as median and interquartile range (see SFig. 1A-C). These effect modifiers showed no considerable difference among the treatments. We, therefore, assumed that there was no intransitivity of the data.

Risk of bias in individual trials. All included trials applied the randomization study design (see SFig. 2A). Except for one trial ${ }^{32}$, nine ones implemented the double-blindness method. Of these, one trial did not describe the randomization process and the concealment of allocation ${ }^{8}$. These two trials were, therefore, rated as trials with a high risk of bias. Two trials had some concerns on the methods used to ensure the allocation concealment, which resulted in the trial rating of a moderate risk of bias ${ }^{6,7}$.

Results of individual trials. Compared to placebo, the efficacy of lurasidone in reducing overall psychotic symptoms was as follows: (1) lurasidone $20 \mathrm{mg} /$ day was not significantly effective in two trial ${ }^{8,33}$, (2) lurasidone 


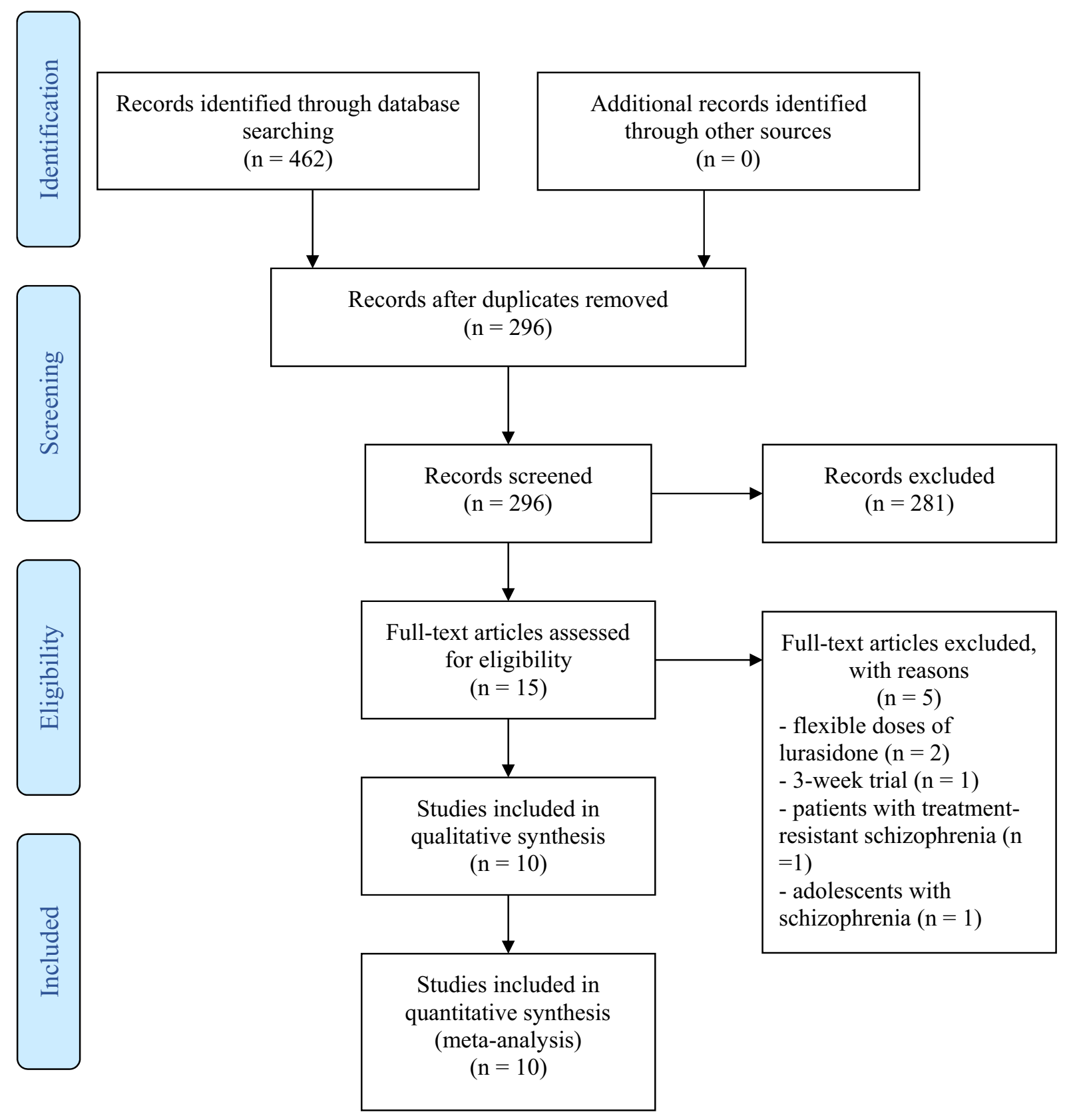

Figure 1. PRISMA flow diagram: records retrieved from database searches and trial inclusion in the systematic review and network meta-analysis of lurasidone doses for schizophrenia.

$40 \mathrm{mg} /$ day was significantly effective in one ${ }^{35}$ but not in five trials ${ }^{6-9,37}$, (3) lurasidone $80 \mathrm{mg} /$ day was significantly effective in three ${ }^{9,34,36}$ but not in the other three trials ${ }^{6-8}$, (4) lurasidone $120 \mathrm{mg} /$ day was significantly effective in two ${ }^{35,37}$ but not in one trial ${ }^{9}$, and (5) lurasidone $160 \mathrm{mg} /$ day was significantly effective in one trial ${ }^{34}$ (see Table 1).

Most trials reported the dichotomous data of somnolence, EPS, adverse dropout, and all-cause dropout rates, but only one trial reported that all-cause dropout rates between lurasidone $80 \mathrm{mg} /$ day and placebo were not significantly different ${ }^{36}$. Other treatment outcomes reported in individual trials can be found in Table 1.

Synthesis of results. All continuous outcomes were assessed using the same scales and, therefore, aggregated using the (weighted) mean differences (MDs) of scale scores. The scales used were as follows: PANSS total scores for the severity of overall psychotic symptoms, PANSS positive scores for the severity of positive symptoms, PANSS negative scores for the severity of negative symptoms, MADRS scores for the severity of depression symptoms, and kilograms for weight gain.

Figure $3 \mathrm{~A}$ shows the forest plot of pooled MDs comparing PANSS total score reduction between five lurasidone doses and placebo. Except for lurasidone $20 \mathrm{mg} / \mathrm{day}$, lurasidone 160, 120, 80, and $40 \mathrm{mg} /$ day was significantly superior to placebo. Among these four doses, lurasidone $160 \mathrm{mg} /$ day was the most effective $(\mathrm{MD}=-13.46$, $95 \% \mathrm{CI}:-19.97$ to -6.95$)$. Table $2 \mathrm{~A}$ shows that lurasidone $160 \mathrm{mg} /$ day significantly outperformed lurasidone 120 , 


A: PANSS total score - 10 trials, 10
treatments, and 43 pairwise
comparisons

Figure 2. Network plots of five different doses of lurasidone, other antipsychotics, and placebo for acute schizophrenia. The nodes in the graph layout correspond to lurasidone at various doses, other antipsychotics, and placebo. Lines display the observed treatment comparisons. The thickness of edge indicates the number of comparisons. Gray/blue areas indicate the availability of trials with three doses or more. PANSS: Positive and Negative Syndrome Scale; MADRS: Montgomery-Asberg Depression Rating Scale; EPS: extrapyramidal symptoms. HAL: Haloperidol; LU20: Lurasidone $20 \mathrm{mg}$ /day; LU40: Lurasidone $40 \mathrm{mg} /$ day; LU80: Lurasidone 80 mg/day; Lurasidone 120 mg/day; Lurasidone 160 mg/day; OLA: Olanzapine; PLA: Placebo; QUE: Quetiapine; RIS: Risperidone.

80,40 , and $20 \mathrm{mg} /$ day. Lurasidone $80 \mathrm{mg} /$ day ranked higher than but was not significantly superior to lurasidone $120 \mathrm{mg} /$ day $(\mathrm{MD}=-0.59,95 \% \mathrm{CI}:-5.10$ to 3.93$)$.

Figure $3 \mathrm{~B}$ shows the forest plot of pooled RRs comparing all-cause dropout rates associated with five lurasidone doses and placebo. Lurasidone $160 \mathrm{mg} /$ day and $80 \mathrm{mg} /$ day were associated with significantly fewer rates of all-cause dropouts compared to placebo $(\mathrm{RR}=0.63,95 \% \mathrm{CI}=0.43$ to 0.91 and $\mathrm{RR}=0.87,95 \% \mathrm{CI} 0.77$ to 0.99 , respectively). All-cause dropout rates among five lurasidone doses were not significantly different (see Table 2B).

The forest plots and league tables of other outcomes can be found in SFig. 3 and STable 3. The treatment effects on PANSS positive scores was similar to that of PANSS total scores (see SFig. 3A and STable 3A). For PANSS negative score reduction, lurasidone $160,120,80$, and $40 \mathrm{mg}$ /day were significantly superior to placebo, but these four doses were not significantly different among each other (see SFig. 3B and STable 3B). Together with lurasidone $160 \mathrm{mg} /$ day, lurasidone $80 \mathrm{mg} /$ day also significantly outperformed lurasidone $120 \mathrm{mg} /$ day $(\mathrm{MD}=-1.86$, $95 \% \mathrm{CI}:-2.99$ to -0.73 ) and placebo in reducing MADRS score (see SFig. 33 and STable 3C). Weight gain associated with lurasidone 80 and $40 \mathrm{mg} /$ day was significantly higher than that with placebo (see SFig. 3D and STable 3D). Somnolence related to lurasidone $160,120,80$, and $40 \mathrm{mg} /$ day was significantly more common than that to placebo (see SFig. 3E and STable 3E). The EPS rates associated with lurasidone 120 and $40 \mathrm{mg} /$ day were significantly higher than that related to placebo (see SFig. $3 \mathrm{~F}$ and STable $3 \mathrm{~F}$ ). The adverse dropout rates associated with lurasidone $160,120,80$, and $40 \mathrm{mg}$ /day were not significantly higher than that related to placebo (see SFig. 3G and STable 3G).

Heterogeneity and inconsistency. Among the nine outcomes, global heterogeneity (incoherence) was a major concern only for the dataset of PANSS total score reduction $\left(\chi^{2}=26.83, \mathrm{df}=14, \mathrm{p}=0.02\right)$ (Fig. 3A). There was no concern about the global heterogeneity of data related to all-cause dropout rates $(\chi 2=13.92, d=14$, $p=0.46$ ) (Fig. $3 \mathrm{~B})$ and the data of the other seven outcomes $(p>0.10)$ (see SFig. 3A-G). Major concerns and some concerns for the local inconsistency can be found in STable 4A-I.

Risks of bias across studies and additional analysis. SFigure $2 \mathrm{~B}$ shows the risks of bias across trials weighted by the sample sizes. The high risks of bias in two domains and the overall risk of bias were less than $25 \%$. SFig. 4A-I show the funnel plots for exploring the publication bias of nine outcomes. Of these, significant publication biases were found in the datasets of weight gain and adverse dropout rates (Egger's test $p<0.01$ and $\mathrm{p}=0.01$, respectively). 


\begin{tabular}{|c|c|c|c|c|c|}
\hline Trial (year) & Duration (weeks) & Lurasidone dose (mg/day) & Control/comparator (mg/day) & Patient population & $\begin{array}{l}\text { Available outcomes of interest } \\
\text { (measures)-results b,c }\end{array}$ \\
\hline Nakamura 2009 & 6 & $80 \mathrm{mg} /$ day $(n=90)$ & Placebo $(n=90)$ & $\begin{array}{l}\text { Schizophrenia, acute exacerbation } \\
\text { of symptoms; age } 18-64 \text { years }\end{array}$ & $\begin{array}{l}\text { Overall psychotic symptoms } \\
\text { (PANSS total score): LU80 > PLA } \\
\text { Positive symptoms (PANSS positive } \\
\text { score): LU80 > PLA } \\
\text { Negative symptoms (PANSS nega- } \\
\text { tive score): LU80 > PLA } \\
\text { Depression (MADRS score): } \\
\text { LU80 > PLA } \\
\text { Weight gain: N/A } \\
\text { Somnolence rates: N/A } \\
\text { Adverse dropout rates: LU80 } \approx \text { PLA } \\
\text { All-cause dropout rates: } \\
\text { LU80 } \approx \text { PLA }\end{array}$ \\
\hline Meltzer 2011 & 6 & $\begin{array}{l}40 \mathrm{mg} / \mathrm{day}(n=120) \\
120 \mathrm{mg} / \mathrm{day}(n=119)\end{array}$ & $\begin{array}{l}\text { Placebo }(n=116) \\
\text { Olanzapine } 15 \mathrm{mg} / \text { day }(n=123)\end{array}$ & $\begin{array}{l}\text { Schizophrenia, acute exacerbation } \\
\text { of symptoms; age } 18-75 \text { years }\end{array}$ & $\begin{array}{l}\text { Overall psychotic symptoms } \\
\text { (PANSS total score): LU40, LU120, } \\
\text { OLA > PLA } \\
\text { Positive symptoms (PANSS positive } \\
\text { score): LU40, LU120, OLA >PLA } \\
\text { Negative symptoms (PANSS } \\
\text { negative score): LU40, LU120, } \\
\text { OLA > PLA } \\
\text { Depression (MADRS score): } \\
\text { OLA > PLA } \\
\text { Weight gain: LU40, LU120 } \approx \text { PLA; } \\
\text { OLA > PLA } \\
\text { Somnolence rates: N/A } \\
\text { EPS rates: N/A } \\
\text { Adverse dropout rates: N/A } \\
\text { All-cause dropout rates: N/A }\end{array}$ \\
\hline Loebel 2013 & 6 & $\begin{array}{l}80 \mathrm{mg} / \text { day }(n=125) \\
160 \mathrm{mg} / \text { day }(n=121)\end{array}$ & $\begin{array}{l}\text { Placebo }(n=122) \\
\text { Quetiapine } 600 \mathrm{mg} / \text { day }(n=120)\end{array}$ & $\begin{array}{l}\text { Schizophrenia, acute exacerbation } \\
\text { of symptoms; age } 18-75 \text { years; }\end{array}$ & $\begin{array}{l}\text { Overall psychotic symptoms } \\
\text { (PANSS total score): LU80, LU160, } \\
\text { QUE > PLA } \\
\text { Positive symptoms (PANSS positive } \\
\text { score): LU80, LU160, QUE > PLA } \\
\text { Negative symptoms (PANSS } \\
\text { negative score): LU80, LU160, } \\
\text { QUE > PLA } \\
\text { Depression (MADRS score): LU80, } \\
\text { LU160, QUE > PLA } \\
\text { Weight gain: LU80, QUE > PLA } \\
\text { Somnolence rates: N/A } \\
\text { EPS rates: N/A } \\
\text { Adverse dropout rates: N/A } \\
\text { All-cause dropout rates: N/A }\end{array}$ \\
\hline Nasrallah 2013 & 6 & $\begin{array}{l}40 \mathrm{mg} / \text { day }(n=125) \\
80 \mathrm{mg} / \mathrm{day}(n=123) \\
120 \mathrm{mg} / \text { day }(n=124)\end{array}$ & Placebo $(n=128)$ & $\begin{array}{l}\text { Schizophrenia, acute exacerbation } \\
\text { of symptoms; age } 18-75 \text { years }\end{array}$ & 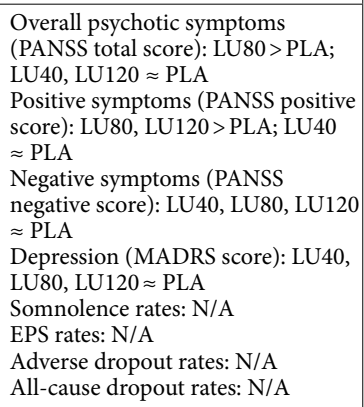 \\
\hline Ogasa 2012 & 6 & $\begin{array}{l}40 \mathrm{mg} / \text { day }(n=50 \\
120 \mathrm{mg} / \text { day }(n=49)\end{array}$ & Placebo $(n=50)$ & $\begin{array}{l}\text { Schizophrenia, acute exacerbation } \\
\text { of symptoms; age 18-64 years }\end{array}$ & 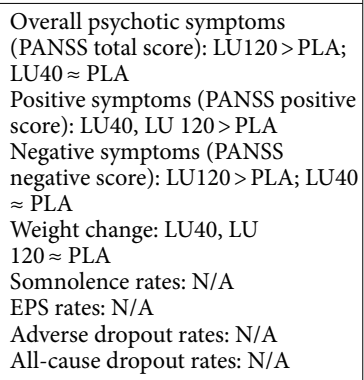 \\
\hline Potkin 2015 & 6 & $\begin{array}{l}20 \mathrm{mg} / \text { day }(\mathrm{n}=71) \\
40 \mathrm{mg} / \mathrm{day}(\mathrm{n}=67) \\
80 \mathrm{mg} / \text { day }(\mathrm{n}=71)\end{array}$ & $\begin{array}{l}\text { Placebo }(n=72) \\
\text { Haloperidol } 10 \mathrm{md} / \text { day }(n=72)\end{array}$ & $\begin{array}{l}\text { Schizophrenia, acute exacerbation } \\
\text { of symptoms; age } 18-64 \text { years }\end{array}$ & $\begin{array}{l}\text { Overall psychotic symptoms } \\
\text { (PANSS total score): LU20, LU40, } \\
\text { LU80, HAL } \approx \text { PLA } \\
\text { Depression (MADRS score): LU20, } \\
\text { LU40, LU80, HAL } \approx \text { PLA } \\
\text { Weight change: N/A } \\
\text { Somnolence rates: N/A } \\
\text { EPS rates: N/A } \\
\text { Adverse dropout rates: N/A } \\
\text { All-cause dropout rates: N/A }\end{array}$ \\
\hline \multicolumn{6}{|l|}{ Continued } \\
\hline
\end{tabular}




\begin{tabular}{|c|c|c|c|c|c|}
\hline Trial (year) & Duration (weeks) & Lurasidone dose (mg/day) & Control/comparator (mg/day) & Patient population & 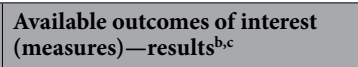 \\
\hline Loebel $2016^{\mathrm{d}}$ & 6 & $20 \mathrm{mg} /$ day $(\mathrm{n}=112$ & Placebo $(n=101)$ & $\begin{array}{l}\text { Schizophrenia, acute exacerbation } \\
\text { of symptoms; age } 18-75 \text { years }\end{array}$ & $\begin{array}{l}\text { Overall psychotic symptoms } \\
\text { (PANSS total score): LU20 } \approx \text { PLA } \\
\text { Weight change: LU20 } \approx \text { PLA } \\
\text { Somnolence rates: N/A } \\
\text { EPS rates: N/A } \\
\text { Adverse dropout rates: N/A } \\
\text { All-cause dropout rates: N/A }\end{array}$ \\
\hline Higuchi 2019a & 6 & $\begin{array}{l}40 \mathrm{mg} / \text { day }(\mathrm{n}=150) \\
80 \mathrm{mg} / \mathrm{day}(\mathrm{n}=155)\end{array}$ & Placebo $(n=152)$ & $\begin{array}{l}\text { Schizophrenia, acute exacerbation } \\
\text { of symptoms; age 18-74 years }\end{array}$ & $\begin{array}{l}\text { Overall psychotic symptoms } \\
\text { (PANSS total score): LU40, LU80 } \\
\approx \text { PLA } \\
\text { Positive symptoms (PANSS positive } \\
\text { score): LU40 PLA; LU80 > PLA } \\
\text { Negative symptoms (PANSS nega- } \\
\text { tive score): LU40, LU80 } \approx \text { PLA } \\
\text { Weight change: LU40, LU80 } \approx \text { PLA } \\
\text { Somnolence rates: N/A } \\
\text { Adverse dropout rates: N/A } \\
\text { All-cause dropout rates: N/A }\end{array}$ \\
\hline Higuchi 2019b & 6 & $\begin{array}{l}40 \mathrm{mg} / \text { day }(\mathrm{n}=125) \\
80 \mathrm{mg} / \text { day }(\mathrm{n}=129)\end{array}$ & $\begin{array}{l}\text { Placebo }(n=152) \\
\text { Risperidone } 4 \mathrm{mg} / \text { day }(n=64)\end{array}$ & Schizophrenia; age $18-75$ years & $\begin{array}{l}\text { Overall psychotic symptoms } \\
\text { (PANSS total score): LU40, LU80, } \\
\text { RIS } \approx \text { PLA } \\
\text { Positive symptoms (PANSS } \\
\text { positive score): RIS > PLA; LU40, } \\
\text { LU80 } \approx \text { PLA } \\
\text { Negative symptoms (PANSS nega- } \\
\text { tive score): LU40, LU80, RIS } \approx \text { PLA } \\
\text { Adverse dropout rates: N/A } \\
\text { All-cause dropout rates: N/A }\end{array}$ \\
\hline Jena 2019 & 6 & $80 \mathrm{mg} /$ day $(\mathrm{n}=51)$ & Olanzapine $10 \mathrm{mg} /$ day $(n=50)$ & Schizophrenia; age $18-45$ years & $\begin{array}{l}\text { Overall psychotic symptoms } \\
\text { (PANSS total score): OLA > LU80 } \\
\text { Positive symptoms (PANSS positive } \\
\text { score): OLA > LU80 } \\
\text { Negative symptoms (PANSS nega- } \\
\text { tive score): OLA LU80 } \\
\text { All-cause dropout rates: N/A }\end{array}$ \\
\hline
\end{tabular}

Table 1. Characteristics and key results of the included trials ${ }^{\mathrm{a}}$. ${ }^{\mathrm{a}}$ Only the last results of the study. ${ }^{\mathrm{b}}$ The symbols of $>$ and $\approx$ indicate significant superiority $(p<0.05)$ and not significant difference $(p \geq 0.05)$ as being reported by the authors, respectively. ${ }^{\mathrm{N}} \mathrm{N} / \mathrm{A}$ indicates the availability of data but no statistical test being applied. ${ }^{\mathrm{d}}$ Participants initiating with lurasidone $80 \mathrm{mg} /$ day were excluded. Those nonresponding to 2-week treatment of lurasidone $80 \mathrm{mg}$ /day were randomized to further received lurasidone $80 \mathrm{mg} /$ day and lurasidone $160 \mathrm{mg} /$ day. PANSS: Positive and Negative Syndrome Scale; MADRS: Montgomery-Asberg Depression Rating Scale; EPS: extrapyramidal symptoms. LU20, LU40, LU80, LU120, LU160: Lurasidone 20, 40, 80, 120, 160 mg/day, respectively. $\mathrm{HAL}=$ haloperidol; $\mathrm{OLA}=$ olanzapine; $\mathrm{PLA}=$ placebo; $\mathrm{QUE}=$ quetiapine; and $\mathrm{RIS}=$ risperidone.

Confidence of NMA estimates. Although the literature search retrieved published and unpublished information (e.g., clinictrials.gov), reporting bias of all NMA estimates was suspected because nine of ten trials were industry-funded. This domain, therefore, needed no further suspect related to the publication bias. Indirectness was not an issue of concern because all participants were adults with acute schizophrenia. Moreover, we found no obvious intransitivity of the effect modifiers. For the imprecision, we set the meaningful sizes of effect for the continuous outcomes as follows: (1) a 15-point reduction of PANSS total scale ${ }^{38}$, (2) a 3.5-point reduction of 7-item PANSS positive or negative subscale, (3) 2-point reduction of MADRS ${ }^{39}$ and (4) $0.9 \mathrm{~kg}$ of weight gain. The 15-point reduction of 30-item PANSS total scale was used to proportionally set the 3.5-point reduction of 7-item PANSS positive or negative subscale. In a large RCT, olanzapine increased the body weight of patients with schizophrenia by an average of $0.9 \mathrm{~kg} /$ month $^{40}$. These cut-offs were used to set the imprecision of NMA estimates. STable 5A-I shows the risk-of-bias charts of all outcomes.

After implementing the judgments and calculations on the issues mentioned above, all NMA estimates were rated as low and very low confident (see STable 5A-I). The low and very low confidence levels were mainly caused by the suspect of reporting bias, which resulted in rating down the confidence by two levels from high to low levels. Table 3A,B and STable 6A-G show the confidence rating and effect estimates of each comparison among lurasidone doses and placebo.

Dose-response relationships. Four doses of $160,120,80$, and $40 \mathrm{mg}$ /day were included in the doseresponse analyses of their interactions to positive and negative symptoms. For the other six outcomes, five doses of $160,120,80,40$, and $20 \mathrm{mg} /$ day were involved in the analyses.

Figure 4 shows the dose-response curves and ED50 of eight outcomes derived from the NMA estimates comparing lurasidone at different doses and placebo. Together with the nonexistence of plateau, the ED50's of lurasidone for PANSS total, PANSS positive, and MADRS score reductions were higher than $80 \mathrm{mg} /$ day. For the rest outcomes, the plateaus could be observed, and the ED50's were lower than $80 \mathrm{mg} /$ day. 


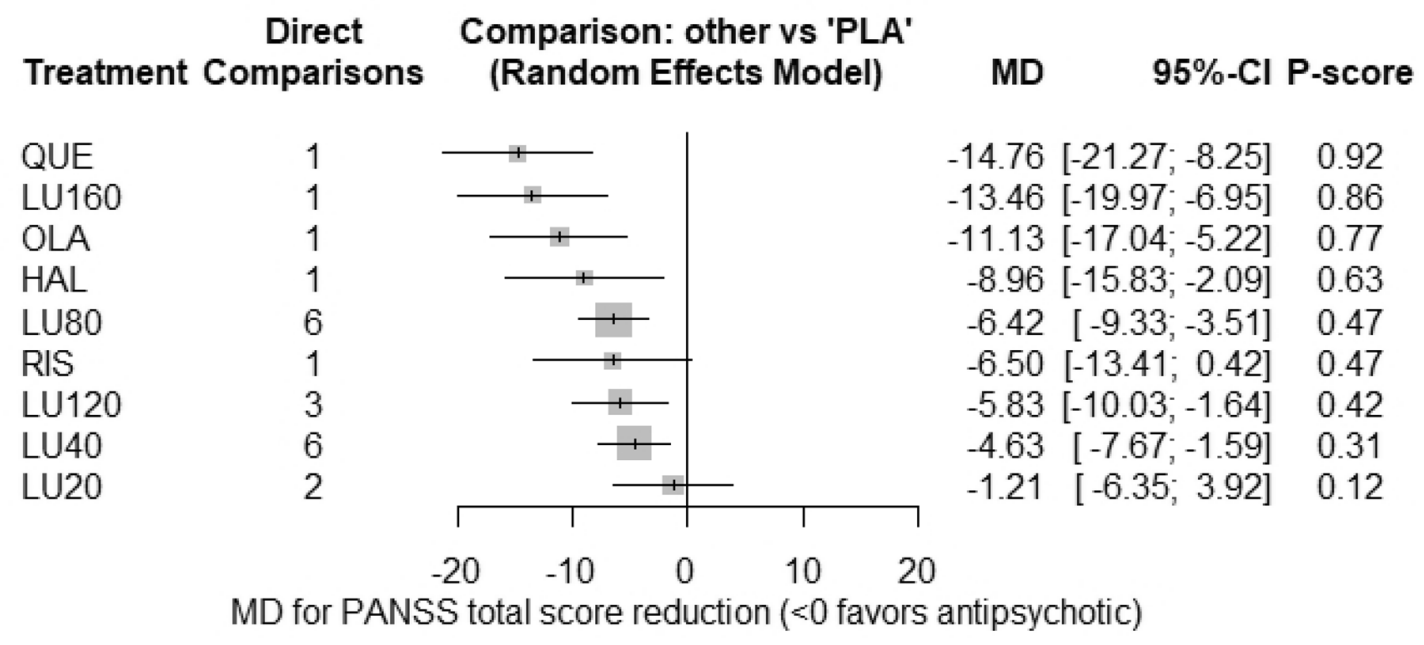

(A) PANSS total score reduction (Heterogeneity: $\chi 2=26.83, \mathrm{df}=14, \mathrm{p}=0.02$ )

$\begin{array}{lr}\text { Direct } \\ \text { Treatment Comparisor } \\ \text { QUE } & 1 \\ \text { RIS } & 1 \\ \text { LU160 } & 1 \\ \text { OLA } & 1 \\ \text { LU80 } & 6 \\ \text { LU40 } & 6 \\ \text { LU120 } & 3 \\ \text { LU20 } & 2 \\ \text { HAL } & 1\end{array}$

Comparison: other vs 'PLA'

(Random Effects Model)

RR $\quad 95 \%$-Cl P-score

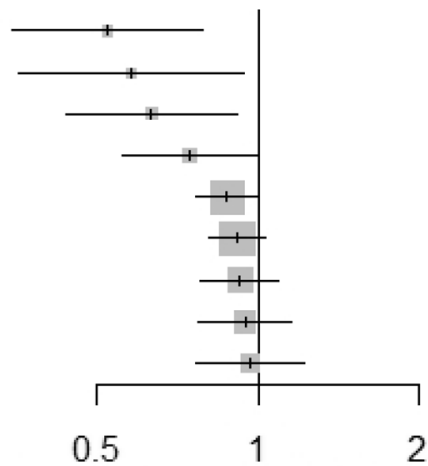

$0.52[0.35 ; 0.79] \quad 0.92$

$0.58[0.36 ; 0.94] \quad 0.84$

$0.63[0.43 ; 0.91] \quad 0.80$

$0.74[0.55 ; 1.00] \quad 0.67$

$0.87[0.77 ; 0.99] \quad 0.47$

$0.91[0.81 ; 1.04] \quad 0.35$

$0.92[0.78 ; 1.09] \quad 0.32$

$0.94[0.77 ; 1.15] \quad 0.28$

$0.96[0.76 ; 1.22] \quad 0.24$

RR for all-cause dropout rates ( $<1$ favors antipsychotic)

(B) All-cause dropout rates (Heterogeneity: $\chi 2=13.92, \mathrm{~d}=14, \mathrm{p}=0.46$ )

Figure 3. Forest plots: the efficacy and acceptability of lurasidone at different doses and other antipsychotics compared to placebo and the global heterogeneity (inconsistency) of data. MD: mean difference; RR: relative risk; P-score: p-value for treatment ranking. PANSS: Positive and Negative Syndrome Scale; LU20, LU40, LU80, LU120, LU160: Lurasidone 20, 40, 80, 120, $160 \mathrm{mg}$ /day, respectively. HAL = haloperidol; OLA = olanzapine; $\mathrm{PLA}=$ placebo; $\mathrm{QUE}=$ quetiapine; and RIS $=$ risperidone .

\section{Discussion}

Lurasidone 160, 120, 80, and $40 \mathrm{mg} /$ day are effective for treating acute schizophrenia, and the dose of $160 \mathrm{mg} /$ day outperforms the other three doses. Only two doses of 160 and $80 \mathrm{mg} /$ day are associated with fewer dropouts and can reduce depression symptoms. The findings of ED50's larger than $80 \mathrm{mg} /$ day suggest that the maximal effective doses for PANSS total score, PANSS positive score, and MADRS score reductions might be higher than 


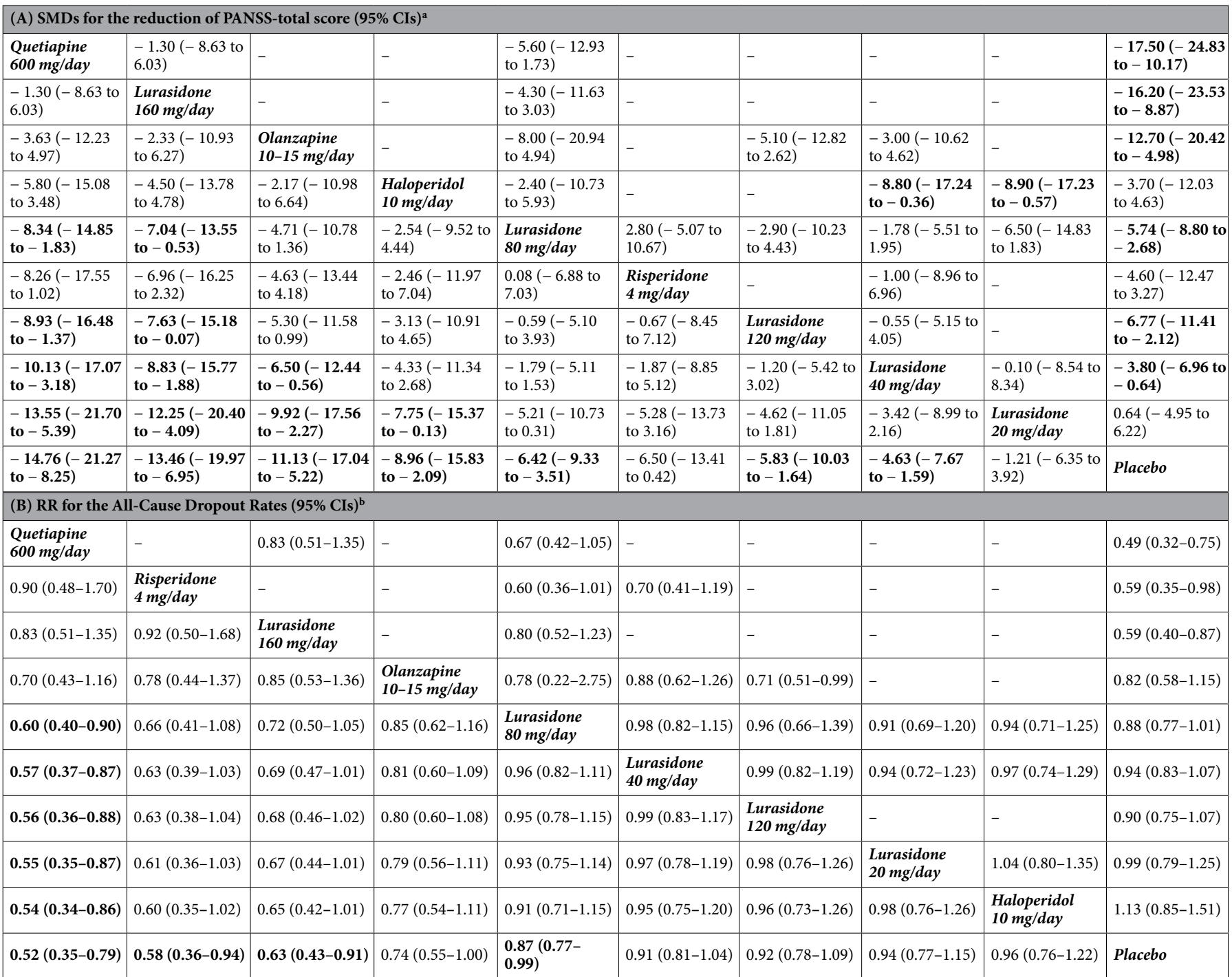

Table 2. League tables presenting network meta-analysis estimates: (lower triangle) and direct estimates (upper triangle): primary efficacy and acceptability outcomes of lurasidone at different doses and placebo for schizophrenia. ${ }^{a} \mathrm{~A}$ mean difference (MD) less than 0 indicates the superiority of treatment defined in the column over the other treatment defined in the row. ${ }^{\mathrm{b}}$ An odd ratio (OR) less than 1 indicates the fewer events of treatment defined in the column over the other treatment defined in the row. - indicates the nonavailability of direct estimate. Treatments are reported in order of ranking of efficacy. Comparison treatments should be read from left to right, and the MD or RR is in the cell in common between the column-defining treatment and the row-defining treatment. A bold estimate indicates the significant difference between the treatment pair.

$160 \mathrm{mg} /$ day. However, lurasidone might produce the maximum effects for negative symptoms and the other four adverse outcomes at the doses between 40 and $160 \mathrm{mg} /$ day.

The present findings are in line with those of a PMA ${ }^{5}$. Our NMA estimates confirm the efficacy of lurasidone $40-160 \mathrm{mg} /$ day for mitigating overall psychotic and positive symptoms of schizophrenia. The similar adversedropout rates of lurasidone $40-160 \mathrm{mg} /$ day and placebo suggest the mild adverse effects of lurasidone. During the 6-week treatment of lurasidone, the patients may gain weight for $0.61 \mathrm{~kg}$ or less. Lurasidone $40-160 \mathrm{mg} / \mathrm{day}$ may increase a two- to four-fold risk of somnolence and EPS for two to four fold.

The findings of this study are similar to those of a PMA but not a NMA comparing the different doses of lurasidone. This study confirms previous PMA findings that the higher doses of lurasidone reduce psychotic symptoms to a greater extent and increase the risks of side effects ${ }^{12}$. However, this study also found the plateau of weight gain and adverse dropouts within the dose range of 40-80 mg/day. The present findings did not support the similar efficacy of lurasidone 80 and $40 \mathrm{mg}$ /day reported in a previous $\mathrm{NMA}^{10}$.

Despite the uses of different statistical techniques and datasets, the dose-response relationships of lurasidone for overall psychotic symptoms found in a previous meta-analysis and this study were relatively similar ${ }^{13}$. Leucht and colleagues (2020) conducted their meta-analysis using a multivariate statistical technique to plot both metaanalysis estimates and their $95 \% \mathrm{CIs}^{41}$. However, the present study applied the widely-used NMA techniques 


\begin{tabular}{|c|c|c|c|c|}
\hline Treatment comparisons & $\begin{array}{l}\text { Direct estimate: RR }(95 \% \\
\text { CI) }\end{array}$ & $\begin{array}{l}\text { Indirect estimate: RR } \\
(95 \% \mathrm{CI})\end{array}$ & $\begin{array}{l}\text { NMA estimate: RR }(95 \% \\
\text { CI) }\end{array}$ & Confidence rating \\
\hline \multicolumn{5}{|c|}{ (A) PANSS-total score reduction } \\
\hline LU160 vs. Placebo & $-16.20[-23.53 ;-8.87]$ & $-3.19[-17.37 ; 11.00]$ & $-13.46[-19.97 ;-6.95]$ & Low \\
\hline LU120 vs. Placebo & $-6.77[-11.41 ;-2.12]$ & $-1.72[-11.47 ; 8.03]$ & $-5.83[-10.03 ;-1.64]$ & Low \\
\hline LU80 vs. Placebo & $-5.74[-8.80 ;-2.68]$ & $-12.73[-22.06 ;-3.40]$ & $-6.42[-9.33 ;-3.51]$ & Low \\
\hline LU40 vs. Placebo & $-3.80[-6.96 ;-0.64]$ & $-14.66[-25.63 ;-3.68]$ & $-4.63[-7.67 ;-1.59]$ & Very low \\
\hline LU20 vs. Placebo & $0.64[-4.95 ; 6.22]$ & $-11.22[-24.22 ; 1.78]$ & $-1.21[-6.35 ; 3.92]$ & Low \\
\hline LU160 vs. LU80 & $-4.30[-11.63 ; 3.03]$ & $-17.31[-31.50 ;-3.13]$ & $-7.04[-13.55 ;-0.53]$ & Very low \\
\hline LU160 vs. LU40 & - & $-8.83[-15.77 ;-1.88]$ & $-8.83[-15.77 ;-1.88]$ & Very low \\
\hline LU160 vs. LU20 & - & $-12.25[-20.40 ;-4.09]$ & $-12.25[-20.40 ;-4.09]$ & Very low \\
\hline LU120 vs. LU160 & - & $7.63[0.07 ; 15.18]$ & $7.63[0.07 ; 15.18]$ & Very low \\
\hline LU120 vs. LU80 & $2.90[-4.43 ; 10.23]$ & $-0.83[-6.57 ; 4.91]$ & $0.59[-3.93 ; 5.10]$ & Low \\
\hline LU120 vs. LU40 & $-0.55[-5.15 ; 4.05]$ & $-4.73[-15.42 ; 5.95]$ & $-1.20[-5.42 ; 3.02]$ & Low \\
\hline LU120 vs. LU20 & - & $-4.62[-11.05 ; 1.81]$ & $-4.62[-11.05 ; 1.81]$ & Very low \\
\hline LU40 vs. LU80 & $1.78[-1.95 ; 5.51]$ & $1.83[-5.45 ; 9.11]$ & $1.79[-1.53 ; 5.11]$ & Very low \\
\hline LU20 vs. LU80 & $6.50[-1.83 ; 14.83]$ & $4.19[-3.18 ; 11.57]$ & $5.21[-0.31 ; 10.73]$ & Very low \\
\hline LU20 vs. LU40 & $0.10[-8.34 ; 8.54]$ & $5.99[-1.44 ; 13.42]$ & $3.42[-2.16 ; 8.99]$ & Very low \\
\hline Treatment comparisons & $\begin{array}{l}\text { Direct estimate: RR (95\% } \\
\text { CI) }\end{array}$ & $\begin{array}{l}\text { Indirect estimate: RR } \\
(\mathbf{9 5 \%} \mathrm{CI})\end{array}$ & $\begin{array}{l}\text { NMA estimate: RR (95\% } \\
\text { CI) }\end{array}$ & Confidence rating \\
\hline \multicolumn{5}{|l|}{$\begin{array}{l}\text { (B) For all-cause dropout } \\
\text { rates }\end{array}$} \\
\hline LU160 vs. Placebo & $0.59[0.40 ; 0.87]$ & $1.13[0.36 ; 3.51]$ & $0.63[0.43 ; 0.91]$ & Low \\
\hline LU120 vs. Placebo & $0.90[0.75 ; 1.07]$ & $1.13[0.70 ; 1.82]$ & $0.92[0.78 ; 1.09]$ & Very low \\
\hline LU80 vs. Placebo & $0.88[0.77 ; 1.01]$ & $0.82[0.53 ; 1.26]$ & $0.87[0.77 ; 0.99]$ & Very low \\
\hline LU40 vs. Placebo & $0.94[0.83 ; 1.07]$ & $0.60[0.37 ; 0.98]$ & $0.91[0.81 ; 1.04]$ & Very low \\
\hline LU20 vs. Placebo & $0.99[0.79 ; 1.25]$ & $0.82[0.55 ; 1.21]$ & $0.94[0.77 ; 1.15]$ & Very low \\
\hline LU160 vs. LU80 & $0.80[0.52 ; 1.23]$ & $0.49[0.22 ; 1.10]$ & $0.72[0.50 ; 1.05]$ & Very low \\
\hline LU160 vs. LU40 & - & $0.69[0.47 ; 1.01]$ & $0.69[0.47 ; 1.01]$ & Very low \\
\hline LU160 vs. LU20 & - & $0.67[0.44 ; 1.01]$ & $0.67[0.44 ; 1.01]$ & Very low \\
\hline LU120 vs. LU160 & - & $1.47[0.98 ; 2.20]$ & $1.47[0.98 ; 2.20]$ & Very low \\
\hline LU120 vs. LU80 & $1.05[0.72 ; 1.52]$ & $1.06[0.85 ; 1.33]$ & $1.06[0.87 ; 1.28]$ & Very low \\
\hline LU120 vs. LU40 & $1.01[0.84 ; 1.22]$ & $1.00[0.64 ; 1.57]$ & $1.01[0.85 ; 1.20]$ & Very low \\
\hline LU120 vs. LU20 & - & $0.98[0.76 ; 1.26]$ & $0.98[0.76 ; 1.26]$ & Very low \\
\hline LU40 vs. LU80 & $1.03[0.87 ; 1.21]$ & $1.12[0.83 ; 1.51]$ & $1.05[0.90 ; 1.21]$ & Very low \\
\hline LU20 vs. LU80 & $1.10[0.84 ; 1.45]$ & $1.05[0.75 ; 1.47]$ & $1.08[0.87 ; 1.34]$ & Very low \\
\hline LU20 vs. LU40 & $1.06[0.81 ; 1.40]$ & $0.99[0.71 ; 1.37]$ & $1.03[0.84 ; 1.27]$ & Very low \\
\hline
\end{tabular}

Table 3. Confidence rating and effect estimates of each comparison: lurasidone at different doses vs. placebo for acute schizophrenia. PLA: Placebo; LU20, LU40, LU80, LU120, LU160: Lurasidone 20, 40, 80, 120, 160 mg/ day, respectively.

to compare the treatment effects of the different doses of lurasidone and fitted the dose-response curves using the NMA estimates of the different doses. Although this study could compute both point estimates and their 95\%CIs, only the point estimates from NMA were used for curve fitting. The 95\%CI values were disregarded because the superimposing of three dose-response curves of point estimates and their upper and lower bounds may result in a crossing of these curves, which is difficult for interpretation. Despite of the above-mentioned differences, the ED50 of lurasidone for overall psychotic symptoms (160 mg/day) found in this study still confirms the previous finding that the maximal effective dose of lurasidone for overall psychotic symptoms may be higher than $160 \mathrm{mg} /$ day.

Each schizophrenia syndrome and lurasidone adverse effect has its pattern of doses and responses interaction. The increase of lurasidone doses from $40 \mathrm{mg}$ /day to somewhere higher than $160 \mathrm{mg} /$ day may increase lurasidone therapeutic effects for overall psychotic, positive, and depression symptoms, but not negative symptoms. The increase of lurasidone doses between 40 and $160 \mathrm{mg} /$ day may increase somnolence and ESP severity. Comparison to its product monograph ${ }^{11}$, we did find the plateau of weight gain within the dose range of $40-80 \mathrm{mg} / \mathrm{day}$ but did not find the peak of EPS rates at the dose of $120 \mathrm{mg} /$ day. The current findings would be helpful to guide the dose adjustment of lurasidone for acute schizophrenia.

Our findings support the use of high-dose lurasidone for acute schizophrenia. Lurasidone $160 \mathrm{mg} /$ day is the most effective dose for overall psychotic symptoms and causes all-cause dropouts less than placebo. The high doses also possess higher efficacy for positive and depression symptoms without more severe weight gain and 


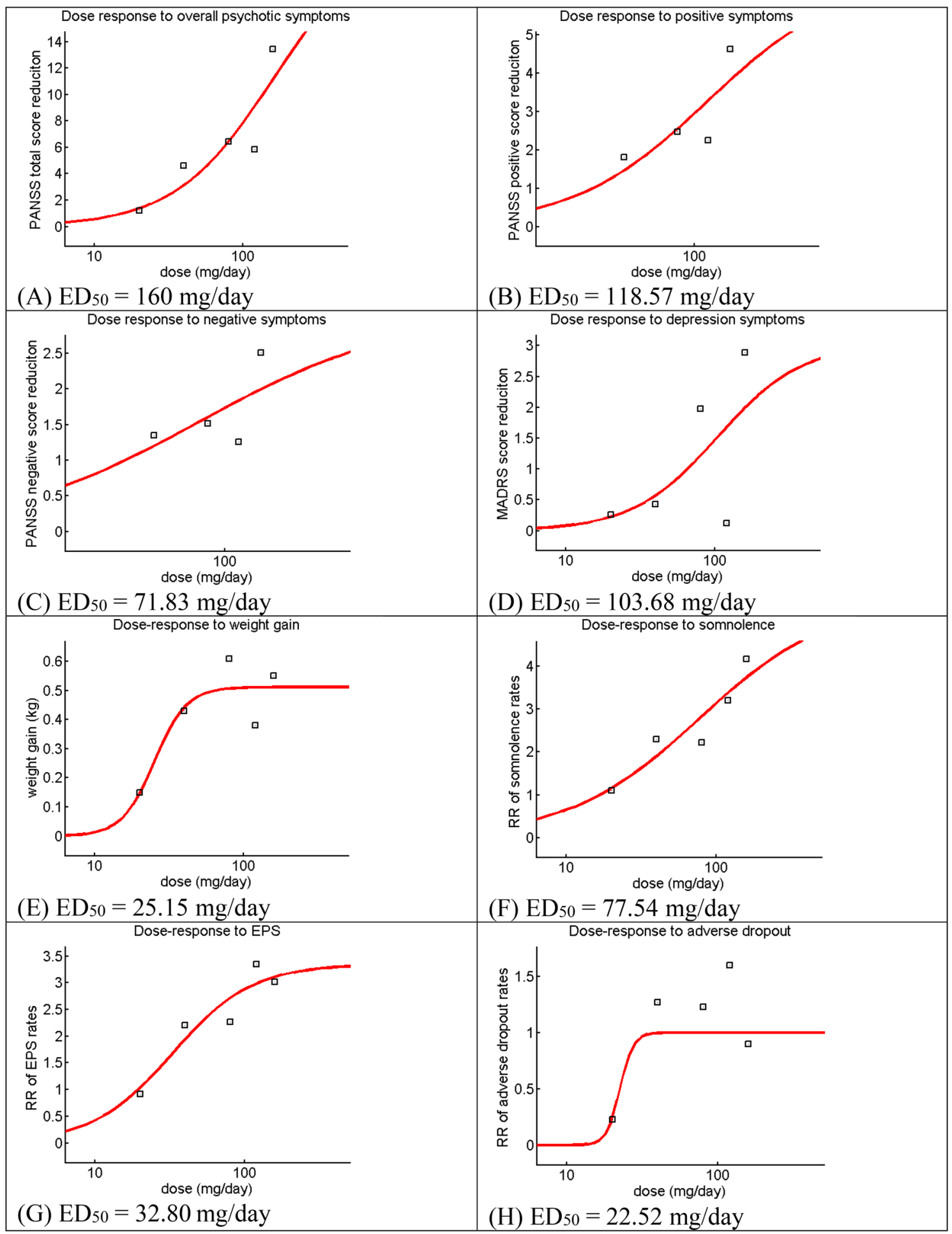

Figure 4. Dose-response curves: therapeutic and adverse effects of lurasidone at different doses for acute schizophrenia. Each curve was plotted with the lurasidone doses on the $\mathrm{x}$-axis and the point NMA estimates of lurasidone against placebo on the y-axis. Figure $4 \mathrm{~A}$ and $4 \mathrm{D}-4 \mathrm{H}$ include five doses of $160,120,80,40$, and $20 \mathrm{mg}$ /day. Figure $4 \mathrm{~B}$ and $4 \mathrm{C}$ include four doses of $160,120,80$, and $40 \mathrm{mg} /$ day. $\mathrm{ED}_{50}=$ half-maximal effective dose, EPS: Extrapyramidal side effects, PANSS: Positive and Negative Syndrome Scale, MADRS: MontgomeryAsberg Depression Rating Scale. 
overall adverse effects than the lower doses. Two drawbacks of the higher doses appear to be the higher risks of somnolence and EPS. In addition, the higher doses give no more benefit for negative symptoms.

Lurasidone doses and effects may not be well correlated. It is not uncommon for a lower dose of an antipsychotic to cause more therapeutic or adverse effects. Despite its non-significance, a dose-response study found that risperidone $6 \mathrm{mg} /$ day was superior to risperidone $10 \mathrm{mg} /$ day in reducing overall psychotic symptoms $\mathrm{s}^{42}$. Likewise, the present study found that lurasidone $80 \mathrm{mg}$ /day significantly outperformed lurasidone $120 \mathrm{mg} /$ day in reducing MADRS score. Together with the findings that lurasidone dosages was not correlated to D2 receptor occupancy ${ }^{43}$, the inconsistent orders of lurasidone doses and effects may need more investigation.

There were some limitations to this NMA. First, the present results should be viewed with caution because the confidence of all NMA estimates was low or very low. Second, some treatments were studied in a small number of patients. For example, the data of lurasidone $160 \mathrm{mg} /$ day, the most effective and acceptable dose, was obtained from 121 adults with acute schizophrenia in a randomized trial only ${ }^{34}$. A type II error, therefore, could not be excluded in the comparison between this dose and others. Third, because the maximum effective doses of lurasidone may be higher than $160 \mathrm{mg} /$ day, the data of dose-response relationships are still incomplete. A trial of lurasidone at the dose of greater than $160 \mathrm{mg} /$ day will add more important evidence for lurasidone dosing. Last, only a few trials of other antipsychotics were included in this NMA. Therefore, we did not have sufficient evidence to compare the pharmacological effects of lurasidone and other antipsychotics.

In conclusions, each schizophrenia syndrome and lurasidone adverse effect has its pattern of doses and responses interaction. Lurasidone $160 \mathrm{mg}$ /day is currently the most effective and acceptable dose for acute schizophrenia, but its maximal effective doses may be higher than $160 \mathrm{mg} /$ day. A trial comparing lurasidone 80 , 120,160 , and more than $160 \mathrm{mg} /$ day is warranted.

\section{Data availability}

The data and $\mathrm{r}$ code of this work are available at https://osf.io/qtn28/.

Received: 9 November 2020; Accepted: 22 February 2021

Published online: 10 March 2021

\section{References}

1. Owen, M. J., Sawa, A. \& Mortensen, P. B. Schizophrenia. Lancet 388, 86-97 (2016).

2. Arvanitis, L. A. \& Miller, B. G. Multiple fixed doses of 'Seroquel' (quetiapine) in patients with acute exacerbation of schizophrenia: A comparison with haloperidol and placebo. The Seroquel Trial 13 Study Group. Biol. Psychiatry 42, 233-246 (1997).

3. Kane, J. M. et al. Efficacy and safety of aripiprazole and haloperidol versus placebo in patients with schizophrenia and schizoaffective disorder. J. Clin. Psychiatry 63, 763-771 (2002).

4. Huhn, M. et al. Comparative efficacy and tolerability of 32 oral antipsychotics for the acute treatment of adults with multi-episode schizophrenia: a systematic review and network meta-analysis. Lancet 394, 939-951 (2019).

5. Zheng, W. et al. Short-term efficacy and tolerability of lurasidone in the treatment of acute schizophrenia: A meta-analysis of randomized controlled trials. J. Psychiatr. Res. 103, 244-251 (2018).

6. Higuchi, T. et al. Lurasidone in the treatment of schizophrenia: Results of a double-blind, placebo-controlled trial in Asian patients. Asia-Pac. Psychiatry 11, e12352 (2019).

7. Higuchi, T. et al. Randomized, double-blind, placebo, and risperidone-controlled study of lurasidone in the treatment of schizophrenia: Results of an inconclusive 6-week trial. Asia Pac. Psychiatry 11, e12354 (2019).

8. Potkin, S. G., Kimura, T. \& Guarino, J. A 6-week, double-blind, placebo- and haloperidol-controlled, phase II study of lurasidone in patients with acute schizophrenia. Ther. Adv. Psychopharmacol. 5, 322-331 (2015).

9. Nasrallah, H. A. et al. Lurasidone for the treatment of acutely psychotic patients with schizophrenia: A 6-week, randomized, placebo-controlled study. J. Psychiatr. Res. 47, 670-677 (2013).

10. Kishi, T., Nosaka, T., Sakuma, K., Okuya, M. \& Iwata, N. Efficacy, tolerability, and safety of lurasidone for acute schizophrenia: A systematic review and network meta-analysis of phase 3 trials in Japan. Neuropsychopharmacol. Rep. 40, 314-322 (2020).

11. Sunovion Pharmaceuticals Canada Inc. Pr Latuda: Product monograph. https://www.sunovion.ca/monographs/latuda.pdf. (2020).

12. Takeuchi, H. et al. Antipsychotic dose in acute schizophrenia: A Meta-analysis. Schizophr. Bull. https://doi.org/10.1093/schbul/ sbaa063 (2020).

13. Leucht, S. et al. Dose-response meta-analysis of antipsychotic drugs for acute schizophrenia. Am. J. Psychiatry 177, 342-353 (2020).

14. Hutton, B. et al. The PRISMA extension statement for reporting of systematic reviews incorporating network meta-analyses of health care interventions: Checklist and explanations. Ann. Intern. Med. 162, 777-784 (2015).

15. Tani, H. et al. Factors associated with successful antipsychotic dose reduction in schizophrenia: A systematic review of prospective clinical trials and meta-analysis of randomized controlled trials. Neuropsychopharmacology 45, 887-901 (2020).

16. Sterne, J. A. C. et al. RoB 2: A revised tool for assessing risk of bias in randomised trials. BMJ 366, 14898 (2019).

17. Krahn, U., Binder, H. \& König, J. A graphical tool for locating inconsistency in network meta-analyses. BMC Med. Res. Methodol. 13, 35 (2013).

18. König, J., Krahn, U. \& Binder, H. Visualizing the flow of evidence in network meta-analysis and characterizing mixed treatment comparisons. Stat. Med. 32, 5414-5429 (2013).

19. R Core Team. R: A Language and Environment for Statistical Computing. (R Foundation for Statistical Computing, Vienna, 2020).

20. RStudio Team. RStudio: Integrated Development Environment for R. (RStudio, Inc., 2020).

21. Rücker, G., Krahn, U., König, J., Efthimiou, O. \& Schwarzer, G. Netmeta: Network Meta-Analysis using Frequentist Methods. (2020).

22. Egger, M., DaveySmith, G., Schneider, M. \& Minder, C. Bias in meta-analysis detected by a simple, graphical test. BMJ 315, 629-634 (1997).

23. Higgins, J. \& Green, S. Cochrane Handbook for Systematic Reviews of Interventions. (The Cochrane Collaboration, 2011).

24. Nikolakopoulou, A. et al. CINeMA: An approach for assessing confidence in the results of a network meta-analysis. PLoS Med. 17, e1003082 (2020).

25. Papakonstantinou, T., Nikolakopoulou, A., Higgins, J. P. T., Egger, M. \& Salanti, G. CINeMA: Software for semiautomated assessment of the confidence in the results of network meta-analysis. Campbell Syst. Rev. 16, e1080 (2020).

26. Di Veroli, G. Y. et al. An automated fitting procedure and software for dose-response curves with multiphasic features. Sci. Rep. 5, 14701 (2015).

27. Feng, Y. et al. Randomized, double-blind, 6-week non-inferiority study of lurasidone and risperidone for the treatment of schizophrenia. Psychiatry Clin. Neurosci. 74, 336-343 (2020). 
28. McEvoy, J. P. et al. Effectiveness of lurasidone in patients with schizophrenia or schizoaffective disorder switched from other antipsychotics: A randomized, 6-week, open-label study. J. Clin. Psychiatry 74, 170-179 (2013).

29. Potkin, S. G., Ogasa, M., Cucchiaro, J. \& Loebel, A. Double-blind comparison of the safety and efficacy of lurasidone and ziprasidone in clinically stable outpatients with schizophrenia or schizoaffective disorder. Schizophr. Res. 132, 101-107 (2011).

30. Goldman, R., Loebel, A., Cucchiaro, J., Deng, L. \& Findling, R. L. Efficacy and safety of lurasidone in adolescents with schizophrenia: a 6-week, randomized placebo-controlled study. J. Child Adolesc. Psychopharmacol. 27, 516-525 (2017).

31. Meltzer, H. Y., Share, D. B., Jayathilake, K., Salomon, R. M. \& Lee, M. A. Lurasidone improves psychopathology and cognition in treatment-resistant schizophrenia. J. Clin. Psychopharmacol. 40, 240-249 (2020).

32. Jena, M. et al. Effect of lurasidone vs olanzapine on neurotrophic biomarkers in unmedicated schizophrenia: A randomized controlled trial. J. Psychiatr. Res. 112, 1-6 (2019).

33. Loebel, A. et al. Lurasidone dose escalation in early nonresponding patients with schizophrenia: A randomized, placebo-controlled study. J. Clin. Psychiatry 77, 1672-1680 (2016).

34. Loebel, A. et al. Efficacy and safety of lurasidone $80 \mathrm{mg} /$ day and $160 \mathrm{mg} /$ day in the treatment of schizophrenia: A randomized, double-blind, placebo- and active-controlled trial. Schizophr. Res. 145, 101-109 (2013).

35. Meltzer, H. et al. Lurasidone in the treatment of schizophrenia: a randomized, double-blind, placebo- and olanzapine-controlled study. Am. J. Psychiatry 168, 957-967 (2011).

36. Nakamura, M. et al. Lurasidone in the treatment of acute schizophrenia: A double-blind, placebo-controlled trial. J. Clin. Psychiatry 70, 829-836 (2009).

37. Ogasa, M., Kimura, T., Nakamura, M. \& Guarino, J. Lurasidone in the treatment of schizophrenia: A 6-week, placebo-controlled study. Psychopharmacology 225, 519-530 (2013).

38. Hermes, E. D. A., Sokoloff, D., Stroup, T. S. \& Rosenheck, R. A. Minimum clinically important difference in the Positive and Negative Syndrome Scale with data from the Clinical Antipsychotic Trials of Intervention Effectiveness (CATIE). J. Clin. Psychiatry 73, 526-532 (2012).

39. Montgomery, S. A. \& Möller, H.-J. Is the significant superiority of escitalopram compared with other antidepressants clinically relevant?. Int. Clin. Psychopharmacol. 24, 111-118 (2009).

40. Lieberman, J. A. et al. Effectiveness of antipsychotic drugs in patients with chronic schizophrenia. N. Engl. J. Med. 353, 1209-1223 (2005).

41. Crippa, A. \& Orsini, N. Dose-response meta-analysis of differences in means. BMC Med. Res. Methodol. 16, 91 (2016).

42. Davis, J. M. \& Chen, N. Dose response and dose equivalence of antipsychotics. J. Clin. Psychopharmacol. 24, 192-208 (2004).

43. Potkin, S. G. et al. D2 receptor occupancy following lurasidone treatment in patients with schizophrenia or schizoaffective disorder. CNS Spectr. 19, 176-181 (2014).

\section{Acknowledgements}

The authors wish to thank Ms.Ruth Leatherman for her assistance in editing the manuscript.

\section{Author contributions}

M.S.: Conceptualization, Data curation, Formal analysis, Investigation, Software, Visualization, Writing-original draft, and Writing - review \& editing. S.S.: Conceptualization, Data curation, Formal analysis, Investigation, Validation, Visualization, Writing — original draft, and Writing—review \& editing. S.L.: Conceptualization, Validation, Writing—original draft, and Writing—review \& editing. N.M.: Conceptualization, Validation, Writingoriginal draft, and Writing—review \& editing. B.M.: Conceptualization, Validation, Writing—original draft, and Writing-review \& editing.

\section{Funding}

This work was supported by Chiang Mai University, Chiang Mai, Thailand (grant no. 16/2563). However, the funding source was not involved in the study design, data collection, data analysis, data interpretation, manuscript preparation, and the decision to submit this article for publication.

\section{Competing interests}

In the last three years, MS has received speaker's honoraria from Lundbeck A/G, Janssen (Thailand), and Sumitomo Dainippon Pharmaceuticals. In the last three years, SS has received speaker's honoraria from Mitsubishi Tanabe Pharma (Thailand) and Sumitomo Pharmaceuticals (Thailand). The other authors have no conflicts of interest.

\section{Additional information}

Supplementary Information The online version contains supplementary material available at https://doi. org/10.1038/s41598-021-84836-Z.

Correspondence and requests for materials should be addressed to M.S.

Reprints and permissions information is available at www.nature.com/reprints.

Publisher's note Springer Nature remains neutral with regard to jurisdictional claims in published maps and institutional affiliations.

(c) (i) Open Access This article is licensed under a Creative Commons Attribution 4.0 International License, which permits use, sharing, adaptation, distribution and reproduction in any medium or format, as long as you give appropriate credit to the original author(s) and the source, provide a link to the Creative Commons licence, and indicate if changes were made. The images or other third party material in this article are included in the article's Creative Commons licence, unless indicated otherwise in a credit line to the material. If material is not included in the article's Creative Commons licence and your intended use is not permitted by statutory regulation or exceeds the permitted use, you will need to obtain permission directly from the copyright holder. To view a copy of this licence, visit http://creativecommons.org/licenses/by/4.0/.

(c) The Author(s) 2021 